\title{
INSCRIPCIONES FUNERARIAS COLECTIVAS DE ÉPOCA ROMANA EN EL DISTRITO DE CASTELO BRANCO (PORTUGAL) ${ }^{1}$
}

\author{
M PILAR GONZÁLEZ-CONDE PUENTE \\ Universidad de Alicante
}

\begin{abstract}
Se estudia aquí un aspecto del hábito epigráfico en las inscripciones del distrito portugués de Castelo Branco, como es la abundancia de enterramientos colectivos, en un número mayor que en el resto de la Península. Se trata de testimonios perfectamente coherentes con el contexto local, tanto en lo que se refiere a los aspectos onomásticos como en el tipo de soporte, y que por su propia estructura permiten rastrear la evolución prosopográfica de los grupos familiares, así como sus modos de inclusión en el ámbito latino.

In this work we deal with a specific aspect of the funerary inscriptions in Castelo Branco (Portugal), in which many collective ones can be found in a higher number than in the rest of the Peninsula. The context is totally local both in onomastic and formal aspects. The structure of these inscriptions helps tracking the onomastic development and its incorporation to the Latin world.
\end{abstract}

La región portuguesa de Beira Baixa presenta en la antigüedad unas peculiaridades que le dan un carácter especial e individualizado del resto de Portugal, y que aparecen reflejadas en la epigrafía romana. Se trata fundamentalmente de diferencias en la onomástica (que no se limitan totalmente a esta zona pero que parecen irradiar de ella) (vid. infra), en los soportes de las inscripciones, en el uso del latín y de las fórmulas habituales, etc. En definitiva, se observa una respuesta particular ante el fenómeno de latinización, que es especialmente sensible en lo que se refiere al proceso de extensión del hábito epigráfico.
Un elemento diferenciador es el grupo de inscripciones destinadas a enterramientos colectivos. Si bien este es un fenómeno habitual en toda la Península, en esta zona alcanza proporciones mayores en relación con el número de epígrafes

\footnotetext{
' La realización de este trabajo no hubiera sido posible sin el generoso apoyo del Prof. J. D'Encarnaçao (Univ. Coimbra), que puso a nuestra disposición el material fotográfico del Museo Lapidario Igaeditano. Nuestra gratitud también a Juan Carlos Olivares (Univ. Alicante), que resolvió algunos de nuestros problemas durante su estancia en Coimbra en otoño de 1997.
} 
conocidos. Aproximadamente 50 inscripciones (del total de 236 de Castelo Branco) responden a esta característica, aunque existen dificultades para definir como piezas destinadas a enterramientos colectivos una serie de epígrafes cuya lectura no conocemos completa, debido al mal estado de conservación del campo epigráfico o bien a la fragmentación de la pieza.

La mayoría de los epígrafes colectivos proceden de Idanha - a - Velha (Idanha - a - Nova) (exactamente 40), dado que allí se da la mayor concentración epigráfica de la zona, y sólo unos pocos de otras localidades, que además se concentran casi en su totalidad en el concelho de Fundao.

Se trata mayoritariamente de inscripciones sobre bloques paralelepipédicos, con el campo epigráfico enmarcado por una moldura, y con el texto repartido en el sentido de su lado mayor, es decir, apaisado. Este tipo de soporte es característico de la epigrafía funeraria de Beira Baixa, y se limita a esta región (D'ENCARNAÇAO 1993, 238, mapa). Los pocos testimonios que transgreden esta norma se encuentran sobre bloques cuadrangulares ( 2 casos), placas de mármol (2 casos), bloque paralelepipédico no moldurado ( 1 caso) y estelas (5 casos).

Las estelas son una cuestión aparte, ya que no tienen relación con el hábito epigráfico local. En un testimonio de Idanha - a - Velha (ALMEIDA 1956, n 29, fig. 117), los difuntos son "lancienses", con una onomástica romana que viene ya de la generación anterior. En cambio, en el epígrafe de Matança (LAMBRINO 1956, 65 s., n ${ }^{\circ} 44=$ AE 1967, 179), la estela parece responder a gustos foráneos pero la onomástica es totalmente indígena y local, incluyendo dos generaciones antes de la de los difuntos, y no deja lugar a dudas sobre lo autóctono del grupo familiar, con nombres como Camira o Arantonius, estrechamente vinculados a la zona de Castelo Branco.

Aparte de estos casos específicos, hay algunos epígrafes de los que sólo conocemos el texto, y no el soporte, porque están en paradero desconocido.

Lo mismo ocurre a la hora de precisar el número de difuntos a los que se ha dedicado la inscripción, ya que en algunos casos no se conserva todo el texto, y sólo podemos intuir que el enterramiento era colectivo, o bien sabemos que lo era pero no se puede precisar el número de los difuntos. Hasta donde podemos saber, hay 31 inscripciones que corresponden al enterramiento de dos personas (una de ellas quizá más), aparte de tres casos dudosos; hay además 13 de tres difuntos, aparte de otro caso dudoso; tres de cuatro difuntos (quizá alguno más teniendo en cuenta las dudas de los de tres ó más); y uno de cinco. Los enterramientos de dos personas son frecuentes, pero es significativo el caso de las colectivas de más de dos.

La relación de los difuntos entre sí es de parentesco próximo, normalmente en primer grado, como es habitual en la epigrafía latina, aunque en algún caso el parentesco no es en primer grado, lo cual no impide dedicar la pieza a varios miembros de la familia, y en menos casos se trata de libertos que comparten patrono. En tres inscripciones de Idanha - a Velha aparecen tres generaciones de una misma familia (vid. infra), que han sido enterradas en la misma tumba. Además es frecuente que el dedicante aproveche tumba y lápida para su propio enterramiento; así, la fórmula et sibi y similares aparece con cierta frecuencia (18 testimonios en enterramientos colectivos).

Aunque todas estas características son habituales en la epigrafía romana, lo cierto es que su frecuencia en esta zona evidencia un esfuerzo por realizar una notable economía de medios. Es frecuente también la dedicación de estas inscripciones por más de un miembro de la familia, generalmente dos, lo que, junto al agrupamiento de los difuntos, facilita el rastreo de la genealogía de los individuos conocidos a través de esta epigrafía funeraria. Con frecuencia, entre dedicantes y difuntos, se menciona a cuatro o más miembros de un grupo familiar.

Este hecho permite, no sólo un estudio onomástico más completo, sino la realización de un análisis del tránsito de la onomástica indígena a una ya más latinizada o plenamente latina. Así tenemos grupos familiares en los que los progenitores tiene todavía onomástica indígena, otros en los que el padre o la madre tienen ya onomástica romana, y otros en los que se aprecia una mayor resistencia a la latinización, manteniendo los hijos nombres indígenas.

Un pequeño grupo de epígrafes (6 casos) representa el mayor núcleo social de resistencia a las nuevas modas onomásticas. Se trata de inscripciones en las que sólo aparece onomástica indígena, toda ella con un marcado carácter local, que se presenta de forma tan reiterativa como es habitual en la zona. Son nombres tan típicos y exclusivos de la región como Camira, Arantonius, Talabus, Cilea, Taganus, Maelo, Camalus, Tapora, Amminius, Catuenus, Toutonus, Boudica, Louius, Cilea (y variantes); junto a ellos, otros nombres indígenas frecuentes en la región, aunque de mayor ámbito geográfico, como Tongius o Tongeta, vinculados familiarmente con los de carácter más local (ABASCAL 1994, passim). Este pequeño grupo no se caracteriza, como podría pensarse, por una cronología más antigua que el resto; más bien puede afirmarse que se trata de fenómenos de no contaminación de la onomástica indígena en unas fechas que, para algunas de las inscripciones, y con la reserva que impone la epigrafía local, alcanzan el siglo II d.C. ${ }^{2}$

\footnotetext{
${ }^{2}$ Sirva de ejemplo una inscripción de Idanha - a - Velha; ALMEIDA 1956, 181, $\mathrm{n}^{\circ}$ 63, fig. 131: Cilea Cili f. / uxsori suae / et Camirae f. su/ae Toutonus Ar/conis f. pater f.c.
} 
El grupo más numeroso es el de las inscripciones con onomástica indígena y romana (27, dos de ellas también con nombres griegos). En la mayoría de los casos se trata de epígrafes en los que aparece la última generación de nombre indígena (los progenitores o al menos uno de ellos), y la primera de nombre latino (los hijos), en los que se plasma ya la aceptación de las nuevas modas onomásticas, pero también la resistencia de algunos nombres indígenas a desaparecer. Todos los epígrafes de este grupo están realizados sobre bloques paralelepipédicos con el texto apaisado, y un buen número de ellas señalan la tumba de más de dos difuntos. La excepción, en lo que al soporte se refiere, es una estela de Idanha (ALMEIDA $1956,223, n^{\circ} 130$ ) que curiosamente contiene nombres locales, y que pertenece a una familia en la que sólo el padre tiene onomástica romana, mientras que el indigenismo de la línea materna se ha mantenido en los hijos.

Por lo que se refiere a los bloques, en una inscripción de Vale Formoso (Covilha) ${ }^{3}$ aparece un grupo familiar que consta aquí de cuatro miembros: el padre, un individuo de nombre latino (Tranquillus), que vuelve a poner a su hijo el nombre de su propio padre4; la madre, que conserva onomástica plenamente indígena; el hijo, que repite nombre de dos generaciones anteriores.; y la hija y dedicante, quien, al igual que su hermano, ostenta un nombre romano de frecuente adopción entre los indígenas".

Un buen ejemplo de la concentración de difuntos con una única dedicación es una inscripción de Idanha (ALMEIDA $1956,204, n^{\circ} 99=$ HAE 1144) en la que una mujer llamada Claudia Tangina dedica a cuatro difuntos con los que le une una relación de parentesco no directa, especificada en el epígrafe, y que la dedicante resuelve finalmente con la fórmula suis f(aciendum) c(urauit). La onomástica, que corresponde a tres generaciones, denota en este caso un contexto plenamente indígena, excepto por lo que se refiere al nomen de la dedicante (Claudia), al que acompaña, sin embargo, un cognomen indígena en una combinación que descubre lo reciente de la adopción de las modas romanas por parte de esta familia.

El resto de los epígrafes presenta una onomástica indígena constantemente reiterada en la zona, como es el caso de

${ }^{3}$ PLÁCIDO 1983, 7 s. (= AE 1983, 473): Tranq(u)illo Silonis f(ilio) patri an/no(rum) L, Siloni Tranq(u)illi f(ilio) fr/atri anno(rum) XV, Boutiae / Mantai f(iliae) matri anno(rum) / XXXXVIII, Auita Tran/q(u)illif(ilia) f(aciendum) c(urauit).

${ }^{4}$ El nombre (Silo) aparece frecuentemente en la zona; se repite en Idanha - a - Velha, y también se encuentra en lugares tan cercanos como Coria y Villar de Plasencia, ambos en la actual provincia de Cáceres (para todos estos testimonios, vid. ABASCAL 1994, 511 s.).

${ }^{5}$ Auita es un nombre frecuente en zonas de indigenismo: PLÁCIDO 1983, 7 s. (= AE 1983, 473).
Arantonius, Cilea, Camala, Taganus, Tanginus (no exclusivo pero sí frecuente); y también otros que aparecen en genitivo por pertenecer a la generación anterior a la responsable del epígrafe, como son Camalus, Amoena, Coelea, Celtiaticus (es frecuente, con sus variantes), Docquirus (ABASCAL 1994, passim).

Otro ejemplo extremo del aprovechamiento lapidario es el de un epígrafe de Idanha (ALMEIDA 1956, 202, n 97, fig. $143=$ HAE 1142) que se dedicó a perpetuar el recuerdo de cuatro difuntos; entre difuntos, dedicante y filiaciones, conocemos a ocho individuos que representan a cuatro generaciones de esta familia: Liguria, hija de Ligur, y Flauus, hijo de Reburrus, son los padres de Sabinus y Camira; ésta, a su vez, casada con Boethus, es madre de Procula. Lo más interesante es el nombre de Camira, que en principio parece poco adecuado para una mujer cuyos padres ostentan nombres romanos (Liguria y Flauus). No es raro en la zona el caso de individuos con nombre romano que repiten en sus hijos la onomástica indígena de sus predecesores (vid. infra).

Otro ejemplo de esta multiplicidad de individuos en una misma inscripción es otro caso de Idanha (ALMEIDA 1956, 215, $\mathrm{n}^{\circ} 116=$ HAE 1157), en donde un individuo llamado Rufus, hijo de Tritius, dedica una inscripción a sus hijos Rufinus y Niger, a su hija Vitalis, y a las que probablemente son sus nueras: Prisca (hija de Fronto y mujer de Niger) y Camala (mujer de Docquirus). Se trata de una familia totalmente romanizada, al menos en lo que se refiere a los gustos onomásticos, que emparenta por matrimonio con una familia de igual grado de latinización (la de Prisca) y con otra de onomástica totalmente indígena. El epígrafe es un buen ejemplo de las diferencias de comportamiento con que una misma generación reacciona a la invasión de lo latino, y de la convivencia de estas diferencias dentro de la sociedad, que no responde a grupos diferenciados.

Un epígrafe de Idanha (ALMEIDA 1956, 219, n 124, fig. $153=$ HAE 1163) fue encargado por una mujer llamada Sila, hija de Silo, para dedicarlo a la memoria de tres miembros de su familia, exactamente su padre, su madre y su tía materna. De nuevo aquí, el aprovechamiento de la pieza afecta no sólo a los parientes próximos, sino a uno de segundo grado, lo cual ya es un hecho menos frecuente. Atendiendo a la onomástica de la familia, la rama materna pertenece a un contexto plenamente indígena y de tradición local (Amoena, Coelea, Maelo... ) mientras que el padre ya lleva nombre romano.

Hay una dedicación funeraria en Idanha (ALMEIDA 1956, 210, no 108, fig. 149) que tiene una estructura algo compleja, especialmente por lo que sus dedicantes quisieron hacer constar. Ofrecida a la memoria de Modestus y de Dutia, se ocuparon de su realización las que respectivamente se denominan mujer e hija, e hija y nieta, de los difuntos. En total, 
entre difuntos, dedicantes y progenitores, son mencionados en la pieza un total de 7 miembros de la familia.

En otro epígrafe de Idanha, una mujer llamada Marcia, hija de Marcius (CIL II 448 = ALMEIDA 1956, 216, nº 119, fig. 152), se ocupó de que un lapidario hiciera una inscripción funeraria para sí misma, para su marido y para su hijo. La dedicante proviene de una familia que ostenta onomástica latina al menos desde una generación anterior, y ha emparentado por matrimonio con una familia que mantiene los gustos indígenas en la onomástica (Reburrus, hijo de Docquirus); en cambio el hijo de ambos ya porta un nombre romano, como su madre (Rufus).

Al igual que la anterior, una mujer llamada Boudica se ocupó, junto con su marido, de que se hiciera una inscripción funeraria para ellos mismos y para su hijo. La dedicante, a pesar de su nombre indígena, es hija de un individuo de nombre romano (Sempronius), lo cual no es extraño en la región, en donde conocemos otros casos de individuos que, por su entorno familiar, deben tener un nomen romano precediendo al cognomen indígena, y que sin embargo no consta en la inscripción; esto supone, al menos, una regresión formal en la onomástica, producida por un hábito local (ALMEIDA 1956, 166, no 38, fig. 122 = HAE 1090); su marido y su hijo portan nombre romano, siendo respectivamente la primera y segunda generación de su familia que cumplen esta condición. Además, sólo aparece en este epígrafe la edad del hijo, y no la de los padres, cosa que ocurre también en la inscripción anterior, debido sin duda a que el epígrafe se encargó en el momento de la muerte del hijo, aunque hay una escasa tradición local de hacer constar la edad de los difuntos.

Más problemas presenta la dedicatoria de Firmus a su familia (ALMEIDA 1956, 172, n $48=$ HAE 1099), ya que el mal estado de conservación de la pieza hace que sólo se pueda leer la parte derecha del texto, que corresponde a la filiación de los difuntos, por lo que están en genitivo: Camalus, Medamus, y probablemente Firmus, nombre tanto del primer difunto como del dedicante e hijo suyo.

Un individuo llamado Domitius también manda hacer un epígrafe colectivo para sus difuntos (LAMBRINO 1956, 60 ss., $\mathrm{n}^{\circ}$ 40; ALMEIDA 1956, 221, nº 127, fig. 155 = HAE 1166; AE 1967, 176). En este caso agrupa a su madre, su padre y su hermana, todos ellos con onomástica indígena local (Taganus, Cilea, Mantaus, Turius); si el dedicante porta onomástica romana, su hermana, en cambio, representa la línea de resistencia a las nuevas modas, a las que los progenitores de ambos no se adaptaron del todo.

Otro ejemplo de inscripción con onomástica indígena y romana es una de Idanha en la que aparecen tres libertas, probablemente las tres difuntas, en cuyo caso no habría dedicante. De una de ellas consta un nomen y un cognomen latinos; la otra menciona un nomen latino y un cognomen indígena, y la tercera mantiene, al menos en la dedicación, sólo el antiguo nombre indígena.

Este grupo presenta una gran uniformidad en la factura de la mayoría de las inscripciones, no sólo en las características de sus soportes, sino incluso en las letras. En cambio, la onomástica romana que aparece en ellas es variada y representativa de diversos monentos, tal y como ocurre con los nomina Cocceius/a, Sempronius, Flauus o Claudia.

Finalmente hay que tener en cuenta un grupo de epígrafes funerarios colectivos cuya onomástica es exclusivamente romana, habiendo desaparecido ya los rastros de indigenismo. Todos ellos proceden de Idanha, excepto uno de Alcaria (Fundao), y todos se encuentran sobre el soporte característico de la zona, es decir, los bloques paralelepipédicos con el texto apaisado; las excepciones son una estela, dedicada a unos difuntos lancienses, que, como todos los inmigrantes de la Meseta Norte, mantienen sus tradiciones originales en lo que a enterramientos se refiere ${ }^{6}$; y dos placas de mármol, una de ellas con moldura decorada que se sale de lo normal dentro de la epigrafía de la región (CIL II 438 y $442=$ ALMEIDA 1956, 159, $\mathrm{n}^{\circ} 26$ e $i d ., 189, \mathrm{n}^{\circ} 76$, fig. 134).

Se puede destacar una inscripción de Alcaria (Fundao) sobre bloque paralelepipédico (INES VAZ 1977, 12 s., nº 6), en la que están representadas cuatro generaciones de una familia, todas ellas con onomástica romana (sucesivamente, en orden cronológico, Sabinus, Sabina y Celsus, Auita y finalmente Claudia Marcea, la dedicante).

Los Sabinii aparecen también en una inscripción funeraria colectiva procedente de Idanha, en la que se vinculan al nomen Cocceius (LAMBRINO 1956, 47 s., nº 23); este gentilicio, escasamente representado en la Península, parecería aportar una fecha post quem para las inscripciones, pero, al menos para las de esta zona, y atendiendo a las características de las piezas, no puede aceptarse este argumento.

Dentro del grupo de inscripciones colectivas con onomástica exclusivamente latina hay sólo dos testimonios de individuos que hagan constar la tribu, en los dos casos la Quirina ${ }^{7}$; se trata de C. Curius Pulli f. Quir. Firmanus (CIL II 442 = ALMEIDA 1956, 189, n 76, fig. 134), y L. Iulius Rufini f. Quir. Fraternus (ALMEIDA 1956, 196, n 88, fig. 139 = HAE 1134; AE 1967, 169; LAMBRINO 1956, 54 s., nº 32). Este último caso desvela tres individuos de una familia de Iulii. También está representado el gentilicio en otra ins-

${ }^{6}$ Sobre la inscripción, ALMEIDA 1956, 160, n² 29, fig. 117 (= HAE 1083). Acerca de los movimientos de población hacia esta zona, vid. HALEY 1986, 185, nº 62.

${ }^{7}$ Acerca de la promoción municipal de la ciudad en época flavia y su pertenencia a la tribu Quirina, vid.: McELDERRY 1918, 73; WIEGELS 1985, 80 s.; ABASCAL y ESPINOSA 1989, 74 s. 
cripción de este mismo grupo ${ }^{8}$, en la que una madre ofrece la dedicatoria para sus hijas (de distinto padre, las dos llevan el nomen materno) y para sí misma. La onomástica recuerda, en el nomen y cognomen, a la de L. Iulius Modestinus (ALMEIDA $1956,198, n^{\circ} 90=$ HAE 1136), y el cognomen lo porta también el munificente $C$. Cantius Modestinus, que dispone de un amplio dosier epigráfico (GIL MANTAS 1988, 227 250).

El grupo de Iulii que conocemos en la ciudad es numeroso, con algunos individuos aislados en el resto del distrito. Tal es el caso de una inscripción de Souto da Casa (Fundao) (SALVADO 1986, 39 ss.; ALVES 1989, 375 s., no 42), en la que aparece una Iulia Modesta, aunque no la misma de Idanha. Todos los individuos que portan este nomen tienen una onomástica totalmente romanizada, hacen constar el tria nomina (y nomen y cognomen en caso de las mujeres) y en algunos casos la tribu. El nomen se asocia a distintos cognomina latinos, como Modestus/Modestinus, Seuerus, etc, y también a nombres griegos en posición de cognomen (SALVADO 1986, 39 ss.) con la excepción de una mujer, Iulia Amoena (ALMEIDA 1956, 200, ${ }^{\circ}$ 93, fig. $141=\mathrm{HAE} 1138$ ), que mantuvo su nombre indígena como cognomen. Además, todos ellos parecen locales, tanto por portar la tribu de la ciudad como por el tipo de soporte que eligen para sus dedicaciones funerarias, que es el propio de la zona y no suele ser aceptado por las familias foráneas. En cambio, no guarda proporción la cantidad de Iulii conocidos con el número de enterramientos colectivos que podemos atribuirles, por lo que no parecen un grupo especialmente proclive a su uso".

Mención aparte merecen dos inscripciones de individuos de origen foráneo, emigrantes de la Meseta Norte. Si bien son enterramientos colectivos, sólo corresponden a dos difuntos por pieza, lo cual es habitual en las inscripciones funerarias romanas y no indica en absoluto un contagio de la costumbre local del aprovechamiento de medios. Una es la estela funeraria de los Anii, lancienses, en la que una mujer dedica a sus dos hijos difuntos (ALMEIDA 1956, 160, n² 29, fig. $117=$ HAE 1083). La otra pieza es un bloque moldurado en el que se ha grabado la inscripción de un cluniense, C. Fabius Vernus, y de su liberta Fabia Bassa (ALMEIDA 1956, 192, n 81 = HAE 1127). Es el unico caso en la región de un inmigrante de la Meseta Norte al que no se entierra con una dedicatoria en estela, que es el modelo culturalmente más cercano

\footnotetext{
* CIL II 444: Iulia Seuerina, Inlia Auita y Iulia Seuera; ALMEIDA 1956, 199, n 92, fig. 140.

'Sólo en dos casos de los estudiados aquí. Sobre el primero: LAMBRINO 1956, 54 s., n 32; ALMEIDA 1956, 196, nº 88, fig. 139; HAE 1134; AE 1967, 169. Sobre el segundo: CIL II 444; ALMEIDA $1956,199, n^{\circ} 92$, fig. 140.
}

a su origen. Al parecer, su heredero así lo decidió, lo que sin duda indica que el difunto no dejó estipulado en su testamento qué tipo de monumento debía señalizar su tumba.

Los habitantes de Beira Baixa aceptaron y asimilaron las formas romanas para perpetuar el recuerdo de sus difuntos, pero se nota entre ellos la falta de una tradición epigráfica funeraria, asimilada antes en otras regiones hispanas. Se aprecia una preocupación por dejar estipuladas las condiciones de su propio enterramiento, tal y como vemos en las fórmulas ex testamento o sibif(aciendum) c(urauit); es decir, se asimila la importancia que los romanos dan a perpetuarse en el tiempo y se adoptan las fórmulas finales en toda su variada gama. Sin embargo, parece que hay una diferencia fundamental en la concepción del monumento. Es cierto que la epigrafía funeraria romana no representa exclusivamente un homenaje individual, en cuanto que el difunto aparece con su filiación y los dedicantes hacen constar expresamente su grado de parentesco con el mismo, es decir, que la dedicación quiere reflejar conscientemente la estructura familiar. Pero en esta región, se refuerza el carácter familiar del monumento funerario, concebido así en su sentido más amplio, como parece derivarse del gran número de enterramientos colectivos, bastantes de ellos con más de dos difuntos, y con frecuencia con varios dedicantes, lo que proporciona un amplio espectro de individuos identificados, mayor que en otras regiones de la Península Ibérica, en relación al número de epígrafes conocidos.

La peculiaridad de los monumentos se aprecia tanto en el soporte como en la inscripción. En el soporte, porque hay uno característico de la zona, los bloques paralelepipédicos, que no aparecen en otras regiones portuguesas, y que es adoptado de forma general por sus habitantes. En cuanto a las inscripciones, se introducen los usos epigráficos y desarrollan una evolución local propia. Las fórmulas y expresiones de la epigrafía no siguen aquí las modas del resto de la Península, sino que se combinan a gusto del usuario de una forma que dificulta la mayoría de las veces la introducción de criterios de datación. Así ocurre por ejemplo con la fórmula inicial D. $M$., que debería haber sido más frecuente en una región en donde el siglo II d.C. proporciona abundante epigrafía. También se encuentran inscripciones con el nombre del difunto en nominativo acompañado de fórmulas más tardías ${ }^{10}$; o bien al contrario, el nombre del difunto en dativo con fórmulas que

\footnotetext{
${ }^{10}$ Valgan como ejemplo estas inscripciones funerarias colectivas:

- LAMBRINO 1956, 41 s., nº 16 (= HAE 1103; AE 1967, 155).

- LAMBRINO 1956, 39 s., nº 13; ALMEIDA 1956, 169, n 43; HAE 1094; AE 1967, 151

- ALMEIDA 1956, 170, n ${ }^{\circ} 45=$ HAE 1096.

- ALMEIDA 1956, 203, nº 98, fig. $144=\mathrm{HAE} 1143$.

- ALMEIDA 1956, 219, n 124, fig. 153 = HAE 1163.
} 
acompañan a nombres en nominativo (ALMEIDA 1956, 202, $n^{\circ}$ 97, fig. $143=$ HAE 1142). Más peculiar resulta un epígrafe (CIL II $448=$ ALMEIDA 1956, 216, ${ }^{\circ}$ 119, fig. 152) en el que el nombre de un difunto está en nominativo y el otro en dativo, y que, atendiendo a sus fórmulas finales, no parece antiguo; es una muestra de lo problemático de aplicar criterios epigráficos generales a la región.

A pesar de las dificultades para la datación, podemos, en principio aceptar unos criterios cronológicos para aquellas inscripciones cuyas características concuerdan entre sí en su totalidad o mayoritariamente. Con estos presupuestos, y por lo que se refiere a las inscripciones funerarias colectivas, puede afirmarse que la aparición de un mayor número de difuntos compartiendo dedicatoria no es aquí indicativo de antigüedad. De las cuatro inscripciones de la zona en las que aparecen más número de difuntos, una tiene los nombres en dativo (LAMBRINO 1956, 55 s., n 33; ALMEIDA 1956, 271, n XVI, fig. 176; HAE 1209; AE 1967, 170), y otras dos unen a esto la fórmula final $f$ (aciendum) $c$ (urauit $)^{\prime \prime}$, por lo que, en ausencia de elementos contradictorios, podemos ubicarlas cronológicamente en el siglo II d.C. ; la cuarta presenta la peculiaridad de expresar los nombres de los difuntos en dativo, pero acompañados de una fórmula funeraria que no le corresponde y que representa un anacronismo que se ha querido mantener a pesar de su pérdida de sentido (h.s.e.s.t.t.l.) (ALMEIDA 1956, 202, $\mathrm{n}^{\circ}$ 97, fig. $143=\mathrm{HAE} 1142$ ).

En definitiva, la epigrafía funeraria romana de Castelo Branco proporciona una visión, no sólo de la composición social de su población, sino también de un aspecto que despierta más interés para este trabajo, cual es el comportamiento de los habitantes de la región frente al fenómeno de latinización, y las diferencias con respecto a las respuestas dadas por población alóctona asentada allí. Este comportamiento conlleva unos usos específicos de las nuevas formas llegadas de Roma que dan como resultado unos testimonios que transgreden continuamente las normas generales de la cotidianeidad romana, y que proporcionan el reto de afrontar su estudio de una forma individualizada y con unos criterios metodológicos distintos. En este contexto, el fenómeno de la epigrafía funeraria colectiva representa una manifestación más de la idiosincrasia regional.

\section{BIBLIOGRAFÍA}

ABASCAL, J.M. 1994: Los nombres personales en las inscripciones latinas de Hispania. Murcia.

ABASCAL, J.M. y ESPINOSA, U. 1989: La ciudad hispano romana. Privilegio y poder. Logroño.

ALMEIDA, F. de. 1956: Egitânia. Lisboa.

ALVES, M.M. 1989: «Para um repertório das inscriçoes romanas do território português (1986)», Euphrosyne 17, pp. 373-384.

D'ENCARNAÇAO, J. 1993: «L'Épigraphie du village à l'extreme occident d'Hispania», en A. Calbi et alii (eds.), L'Epigrafia del Villaggio (Forli 1990). Faenza, pp. 237-259.

GIL MANTAS, V. 1988: «Evergetismo e culto oficial: O constructor de templos C. Cantius Modestinus», en Religio Deorum. Tarragona 1988. Sabadell, pp. 227-250.

HALEY, E.W. 1986: Foreigners in Roman Imperial Spain: investigations of geographical mobility in the spanish provinces of the Roman Empire, 30 B.C. - A.D. 284, Diss. Columbia.

INES VAZ, J.L. 1977: «Inscriçoes romanas no Museu de Fundao», Conimbriga 16, pp. 5-31.

LAMBRINO, S. 1956: «Les inscriptions latines inédites du Musée Leite de Vasconcellos», AP ser. 2, n 3, pp. 5 - 74.

McELDERRY, R.K. 1918: «Vespasian's Reconstruction of Spain», JRS 8, pp. 53-102.

PLÁCIDO, L. 1983: «Placa funerária de Vale Formoso (conv. Scallabitanus)», FE 6, 1983, nº 23.

SALVADO, P. 1986: «Um importante cognomen numa inscriçao de Aldeia do Souto da Casa (Fundao)», Trebaruna 2, pp. 39-41.

WIEGELS, R. 1985: Die Tribusinschriften des römischen Hispanien. Berlín. 